\title{
Interannual Variability and Trends of Extratropical Ozone. Part I: Northern Hemisphere
}

\author{
Xun Jiang, ${ }^{*}+$ Steven Pawson, ${ }^{\#}$ Charles D. Camp, @ J. Eric Nielsen, ${ }^{\# \&}$ Run-Lie Shia,* \\ Ting LiaO, ${ }^{*}$ VARAVut Limpasuvan, ${ }^{+}+$and Yuk L. YunG* \\ * Division of Geological and Planetary Sciences, California Institute of Technology, Pasadena, California \\ \# Global Modeling and Assimilation Office, NASA GSFC, Greenbelt, Maryland \\ @ Mathematics Department, California Polytechnic State University, San Luis Obispo, California \\ \& Science Systems and Applications, Inc., Lanham, Maryland \\ ** Department of Physics, California Institute of Technology, Pasadena, California \\ ++ Department of Chemistry and Physics, Coastal Carolina University, Conway, South Carolina
}

(Manuscript received 31 October 2007, in final form 26 March 2008)

\begin{abstract}
The authors apply principal component analysis (PCA) to the extratropical total column ozone from the combined merged ozone data product and the European Centre for Medium-Range Weather Forecasts assimilated ozone from January 1979 to August 2002. The interannual variability (IAV) of extratropical $\mathrm{O}_{3}$ in the Northern Hemisphere (NH) is characterized by four main modes. Attributable to dominant dynamical effects, these four modes account for nearly $60 \%$ of the total ozone variance in the NH. The patterns of variability are distinctly different from those derived for total $\mathrm{O}_{3}$ in the tropics. To relate the derived patterns of $\mathrm{O}_{3}$ to atmospheric dynamics, similar decompositions are performed for the 30-100-hPa geopotential thickness. The results reveal intimate connections between the IAV of total ozone and the atmospheric circulation.

The first two leading modes are nearly zonally symmetric and represent the connections to the annular modes and the quasi-biennial oscillation. The other two modes exhibit in-quadrature, wavenumber-1 structures that, when combined, describe the displacement of the polar vortices in response to planetary waves. In the $\mathrm{NH}$, the extrema of these combined modes have preferred locations that suggest fixed topographical and land-sea thermal forcing of the involved planetary waves. Similar spatial patterns and trends in extratropical column ozone are simulated by the Goddard Earth Observation System chemistry-climate model (GEOS-CCM).

The decreasing $\mathrm{O}_{3}$ trend is captured in the first mode. The largest trend occurs at the North Pole, with values $\sim-1$ Dobson Unit (DU) $\mathrm{yr}^{-1}$. There is almost no trend in tropical $\mathrm{O}_{3}$. The trends derived from PCA are confirmed using a completely independent method, empirical mode decomposition, for zonally averaged $\mathrm{O}_{3}$ data. The $\mathrm{O}_{3}$ trend is also captured by mode 1 in the GEOS-CCM, but the decrease is substantially larger than that in the real atmosphere.
\end{abstract}

\section{Introduction}

Stratospheric ozone is important because of its climate impacts as well as for its role in shielding the earth from solar radiation. Understanding its decrease as the

+ Current affiliation: Department of Earth and Atmospheric Sciences, University of Houston, Houston, Texas.

Corresponding author address: Yuk L. Yung, Division of Geological and Planetary Sciences, California Institute of Technology, 1200 East California Boulevard, Pasadena, CA 91125.

E-mail: yly@gps.caltech.edu anthropogenic chlorine burden of the stratosphere increased in the late twentieth century and its subsequent recovery as chlorine levels decline is an important task for atmospheric scientists. Attempts to document the decline and recovery of stratospheric ozone are complicated by the role of atmospheric dynamics, which can strongly modulate its interannual variability (IAV; Fusco and Salby 1999; Hood and Soukharev 2003; Randel et al. 2002), and dynamical feedbacks associated with climate change may consequently delay ozone recovery (e.g., Shindell et al. 1999; Austin et al. 2000; Weatherhead et al. 2000). Consequently, ozone recovery is difficult to identify because total column ozone 
exhibits strong IAV, which might be associated with both dynamics and climate change. A primary motivation for studying column ozone IAV is to separate the anthropogenic perturbations of the ozone layer from natural variability (e.g., WMO 2003).

Using rotated principal component analysis, Eder et al. (1999) studied column $\mathrm{O}_{3}$ from 1980 to 1992. They identified 14 contiguous subregions, accounting for more than $70 \%$ of the column ozone variance from $65^{\circ} \mathrm{S}$ to $65^{\circ} \mathrm{N}$. The time series associated with these subregions reveal significant periodicities on semiannual, annual, and quasi-biennial oscillations (QBOs). However, the study did not identify global patterns in column ozone, especially in the polar region, and therefore could not relate the $\mathrm{O}_{3}$ IAV to the annular modes (see later discussion). In a study of the global patterns of ozone variability in the tropics, Camp et al. (2003) showed that $93 \%$ of total column ozone IAV could be explained by four modes whose structures are attributable to the QBO, the interaction between the $\mathrm{QBO}$ and the quasi-biennial oscillation annual beat (QBO-AB), the El Niño-Southern Oscillation (ENSO), and the solar cycle. However, no significant trend was observed in the tropical ozone. In this paper, we investigate $\mathrm{O}_{3} \mathrm{IAV}$ in the polar region for the first time.

Several reasons motivate the extension of the study of Camp et al. (2003) to the extratropics. First, the understanding of how the dominant modes in the tropics (e.g., QBO) propagate to higher latitudes is important but unclear. Second, modes of $\mathrm{O}_{3} \mathrm{IAV}$ in the mid to high latitudes are not well understood. Are they related to the dynamical variability of the atmosphere, including the annular modes (Thompson and Wallace 2000)? Third, the simulation of $\mathrm{O}_{3}$ IAV by $3 \mathrm{D}$ chemistrytransport models is still questionable. Finally, anthropogenic forcing can potentially lead to a significant trend in extratropical ozone. How can we apply this technique to separate the trend from natural variability?

The principal IAV modes of stratospheric ozone and their relationship to dynamical influences can offer clues to improve our understanding of ozone variability and its connection to (and interaction with) climate changes at high latitudes. To date, extensions of principal component analysis (PCA) to the Northern Hemisphere (NH) and Southern Hemisphere ( $\mathrm{SH})$ are hampered by the fact that the most comprehensive merged ozone data (MOD) set (McPeters et al. 1996) lacks $\mathrm{O}_{3}$ data in the polar night region. In addition, as we show, more modes of IAV of $\mathrm{O}_{3}$ exist in the extratropics. Some of these modes are coupled and their physical interpretation is obscure. To resolve some of these difficulties, we report here an innovative study that in- volves four essential components: (i) the use of MOD data supplemented by assimilated data from the European Centre for Medium-Range Weather Forecasts (ECMWF) in the polar night, (ii) the examination of simultaneous analyses of dynamical data relating ozone IAV to the annular modes, (iii) the interpretation the observed ozone IAV using the state-of-the-art Goddard Earth Observing System chemistry-climate model (GEOS-CCM), and (iv) the derivation of the extratropical ozone trend and its confirmation using an independent method, empirical mode decomposition (EMD) analysis (Huang et al. 1998).

The datasets and methodology are described in section 2. The results for PCA of the Northern Hemispheric column ozone and geopotential thickness (GPT) are discussed in section 3. The IAV and trends in model $\mathrm{O}_{3}$ are discussed in section 4. Extended discussions of how our principal findings relate to other studies are presented in section 5. Section 6 contains our conclusions and final remarks. In the appendix, we provide information on the statistical significance of spectral peaks in the power spectrum, cylindrical projections, zonal mean, and $t$ statistics of the leading modes and on the tilting of wavenumber 1 in GPT.

This work is split into two parts. This paper (hereafter Part I) emphasizes the NH. A companion paper (Jiang et al. 2008, hereafter Part II) emphasizes the SH. Extended discussions of how our principal findings from Parts I and II relate to other studies are presented in section 5 of Part II.

\section{Datasets and methodology}

\section{a. Data descriptions}

In this paper, we use four datasets, which are described briefly here. Unless otherwise stated, all data are the monthly mean data.

\section{1) Global total ozone data From OBSERVATIONS}

The focus of this study is the $\mathrm{O}_{3}$ data from the MOD (McPeters et al. 1996), which combines Total Ozone Mapping Spectrometer (TOMS) and Solar Backscattered Ultraviolet (SBUV) satellite datasets. The resolution of the MOD is $5^{\circ}$ latitude $\times 10^{\circ}$ longitude and the regions we studied are from $10-90^{\circ} \mathrm{N}$ or $\mathrm{S}$ for the $\mathrm{NH}$ or SH analysis. Because the MOD data is absent in the polar night, the MOD dataset has been extended to the poles using assimilated $\mathrm{O}_{3}$ and potential vorticity (PV) from the 40-yr ECMWF Re-Analysis (ERA-40) project (Dethof and Holm 2004; Simmons et al. 1999; Uppala et al. 2005). The ECMWF $\mathrm{O}_{3}$ data are pro- 
duced by assimilating satellite observations from January 1979 to December 1988 and from January 1991 to August 2002. Ozone observations by TOMS and SBUV instruments are assimilated in a three-dimensional variational data assimilation (3DVAR) system (Dethof and Holm 2004). Postprocessed data are available on a $2.5^{\circ} \times 2.5^{\circ}$ latitude-longitude grid (Uppala et al. 2005). The MOD and ERA-40 ozone data are very similar where they overlap. The ECMWF $\mathrm{O}_{3}$ has good agreement with the ozonesonde in the polar region after 1979, except that the ECMWF O $_{3}$ at the South Pole is slightly high in October (Dethof and Holm 2004). During 1989 and 1990 when the ECMWF assimilated $\mathrm{O}_{3}$ were not available, ECMWF PV was used as a proxy for $\mathrm{O}_{3}$ based on a mapping method (Manney et al. 1999). Details of validating the ECMWF assimilated $\mathrm{O}_{3}$ and ozonesonde are shown in Fig. A1 in the appendix of Part II.

\section{2) 30-100-HPA GEOPOTENTIAL THICKNESS FROM REANALYSIS}

The 30-100-hPa layer includes the bulk of the ozone layer and has been shown to exhibit strong correlation with ozone column in the tropics (Camp et al. 2003). Geopotential thickness data are used here to demonstrate the connections between ozone variability and atmospheric dynamics. For this work, GPT is computed from geopotential height thicknesses between 30 and $100 \mathrm{hPa}$ from the National Centers for Environmental Prediction (NCEP)-Department of Energy (DOE) Reanalysis 2 (hereafter, NCEP2) (Kalnay et al. 1996; Kistler et al. 2001). The resolution of the data is $2.5^{\circ} \times 2.5^{\circ}$.

\section{3) Ozone From the GEOS-CCM}

Column $\mathrm{O}_{3}$ results from the GEOS-CCM (Stolarski et al. 2006a; Pawson et al. 2008) are used to supplement the analysis of observations in this study. The GEOSCCM is based on the GEOS-4 general circulation model (da Silva et al. 2005) and includes a stratospheric chemistry code (Stolarski et al. 2006b). Coupling between the circulation and chemistry arises through transport and the interactive nature of the radiation code, which allows for the feedback of $\mathrm{O}_{3}, \mathrm{H}_{2} \mathrm{O}, \mathrm{CO}_{2}$, $\mathrm{CH}_{4}, \mathrm{~N}_{2} \mathrm{O}, \mathrm{CFC}-11$, and CFC-12 with the circulation. Archives of monthly mean ozone and meteorological fields were used from a simulation of the period 19512005 at $2^{\circ} \times 2.5^{\circ}$ (latitude-longitude) with 55 layers between the surface and about $80 \mathrm{~km}$. This is run P1 in the study of Pawson et al. (2008), where a more complete model description is given. At the lower boundary, sea surface temperature and sea ice are prescribed from observations compiled by Rayner et al. (2003), along with time-dependent World Meteorological Organization (WMO)-United Nations Environment Programme (UNEP) and Intergovernmental Panel on Climate Change (IPCC) Special Report on Emissions Scenarios (SRES) surface mixing ratios for chemically active and greenhouse gases.

\section{4) Ground-based total ozone at Arosa}

To complement the analysis of the global datasets, we also study the longest record of column $\mathrm{O}_{3}$ data, measured at Arosa $\left(46.8^{\circ} \mathrm{N}, 9.7^{\circ} \mathrm{E}\right)$, Switzerland. (Data can be downloaded from http://www.woudc.org/data_e. html.)

\section{b. Analysis methods}

To extract the spatiotemporal patterns of $\mathrm{O}_{3} \mathrm{IAV}$, we apply PCA (Richman 1986; Preisendorfer 1988; Thompson and Wallace 2000; Camp et al. 2003) to the deseasonalized and weighted column $\mathrm{O}_{3}$ data from MOD, which spans from January 1979 to August 2002. Seasonal cycles for each time series are removed; cycles are determined by taking averages for each month independently.

PCA provides a decomposition of a multivariate dataset into orthogonal spatial patterns, known as empirical orthogonal functions (EOFs), with associated time-dependent amplitude, known as principal component (PC) time series. The EOFs are the eigenfunctions of the covariance matrix of the dataset sorted by the decreasing values of associated eigenvalues. Because these eigenvalues represent the variance captured by each EOF, PCA guarantees that the leading $n$ EOFs capture more of the total variance of the dataset than any other $n$ orthogonal vectors. However, PCA is not a scale-independent method. Therefore, it is necessary to weight each element of the covariance matrix by the area it represents, that is, to scale each time series by the square root of the area, $\sqrt{\cos \theta}$ (where $\theta$ is the latitude), prior to constructing the covariance matrix (Baldwin et al. 2008). The PCA decomposition of the scaled, deseasonalized column-ozone anomaly $\Omega_{A}$ can be represented as

$$
\Omega_{A}(t, \theta, \varphi)=\sum_{i} p_{i}(t) e_{i}(\theta, \varphi)
$$

where $t$ is time, $\varphi$ is longitude, $p_{i}$ is the $i$ th $\mathrm{PC}, e_{i}$ is the $i$ th EOF, and the summation is over all the eigenfunctions with eigenvalues arranged in descending order. To recover the spatial patterns for the original (unscaled) ozone anomaly, we perform a multiple linear regression for each grid point $(\theta, \varphi)$, using as predictors 
TABLE 1. Comparison of the leading modes of variability in column $\mathrm{O}_{3}$ and GPT in the NH.

\begin{tabular}{|c|c|c|c|c|c|}
\hline Modes & 1 & 2 & 3 & 4 & Sum \\
\hline \multicolumn{6}{|c|}{ Fractions of variance } \\
\hline $\mathrm{O} 3$ & $30.4 \%$ & $15.9 \%$ & $7.1 \%$ & $6.5 \%$ & $59.9 \%$ \\
\hline GPT & $32.3 \%$ & $18.5 \%$ & $15 \%$ & $12.4 \%$ & $78.2 \%$ \\
\hline \multicolumn{6}{|l|}{ Spatial pattern } \\
\hline $\mathrm{O} 3$ & Symmetric & Symmetric & Wave 1 & Wave 1 & - \\
\hline GPT & Symmetric & Non-symmetric & Wave 1 & Wave 1 & - \\
\hline \multicolumn{6}{|c|}{ Temporal pattern } \\
\hline 1 & $\mathrm{NAM}+$ trend $3.5 \mathrm{yr}, \mathrm{QBO}, \mathrm{QBO}-\mathrm{AB}, \mathrm{SAO}$ & $\mathrm{BO}, \mathrm{QBO}-\mathrm{AB}, \mathrm{SAO}$ & Stochastic & Stochastic & - \\
\hline 2 & NAM, $3.5 \mathrm{yr}, 17$ months & $\mathrm{BO}, 4-5 \mathrm{yr}, 8 \mathrm{yr}$, trend & Stochastic & Stochastic & - \\
\hline
\end{tabular}

the PC time series $p_{i}(t)$ determined from the scaled PCA of Eq. (1) (Baldwin and Dunkerton 1999; Thompson and Wallace 2000); that is,

$$
\Omega(t, \theta, \varphi)=\sum_{i=1}^{n} C_{i}(\theta, \varphi) p_{i}(t)+R_{n}(\theta, \varphi)
$$

where $\Omega(t, \theta, \varphi)$ represents the deseasonalized (unscaled) $\mathrm{O}_{3}$ anomaly fields. The resulting linear regression coefficients $C_{i}(\theta, \varphi)$ are the spatial patterns of the ozone variability associated with the $i$ th PC time series $p_{i}(t) ; R_{n}$ is the residual of regression. In this study only four modes are used; that is, in Eq. (2), $n=4$. It should be noted that the resulting spatial patterns $C_{i}(\theta, \varphi)$ are no longer mutually orthogonal; the PC time series remain mutually orthogonal.

\section{PCA of Northern Hemispheric column $\mathrm{O}_{3}$ and GPT}

\section{a. Results for Northern Hemispheric column $\mathrm{O}_{3}$}

The four leading modes account respectively for $30.4 \%, 15.9 \%, 7.1 \%$, and $6.5 \%$ of the total variance. Together, they account for $59.9 \%$ of the total variance of $\mathrm{O}_{3}$. Details of the leading modes are listed in Table 1. The spatial patterns of the $\mathrm{O}_{3}$ anomalies regressed upon their leading PCs are shown in Fig. 1. (The polar projection, cylindrical projection, and zonal mean of the regression coefficients are shown in Fig. A2 in the appendix.) The first two modes are relatively zonally symmetric. The first mode represents a hemispheric change in $\mathrm{O}_{3}$, in the sense that if the first principal component $(\mathrm{PC} 1)<0$, ozone is low over the entire $\mathrm{NH}$, with a larger anomaly in the polar region. The second mode has a see-saw structure, describing high $\mathrm{O}_{3}$ in the polar region with low $\mathrm{O}_{3}$ values in the midlatitudes. The third and fourth modes are zonally asymmetric. Their eigenvalues are sufficiently similar that their patterns represent a wavenumber-1 distribution in quadrature.
Physically, the wavenumber-1 structure can be visualized as the wobbling and displacement of the polar vortex in response to planetary waves. Because wave activities are strongest in winter, the results derived from the data in October-March are very similar to those from all data. We also applied the same method to the ECMWF assimilated $\mathrm{O}_{3}$ data; similar results were obtained. Thompson and Wallace (2000) only regressed the column ozone in March (NH) and November (SH) on the standardized monthly time series of the annular modes. In this paper, we study the column ozone data from January 1979 to August 2002 in more detail and reveal that there are symmetric and asymmetric modes in the column ozone that were not apparent in the previous studies.

Further insights into the first four modes are gained by examining the temporal signature in the PCs. The temporal behavior of PC1 is shown in Fig. 2a. For comparison, we overlay the inverted $\mathrm{NH}$ annual mode (NAM) index as a dotted line. The NAM index is the PC associated with the first leading mode, capturing $29 \%$ of the total variance, for the geopotential height at $100 \mathrm{hPa}$ from January 1979 to August 2002 (Baldwin and Dunkerton 1999; Thompson and Wallace 2000). The correlation between the detrended PC1 and the detrended (and inverted) NAM index is 0.53 ( $0.1 \%$ significance level). Linear trends have been removed from PC1 and the inverted NAM index before calculating the correlation. The significance statistics for correlations were generated by a Monte Carlo method (Press et al. 1992; Jiang et al. 2004). A small numerical value of the significance level denotes a high statistical significance. When the polar vortex is stronger (positive NAM index), there is less ozone transported to the polar region (negative PC1) because of a weaker Brewer-Dobson circulation. In addition, a stronger polar vortex with lower temperatures will also result in stronger chemical ozone loss. The El Chichón and Pinatubo eruptions happened in April 1982 and June 1991, respectively. The PC1 values are negative after 
(a)

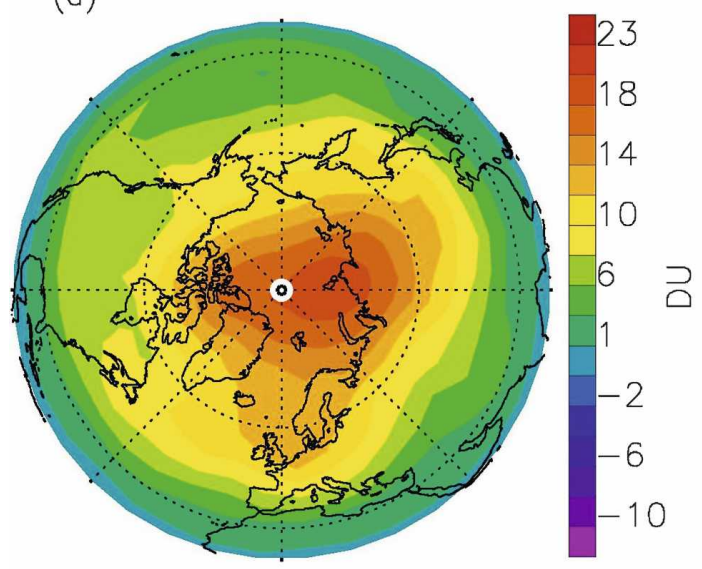

(c)

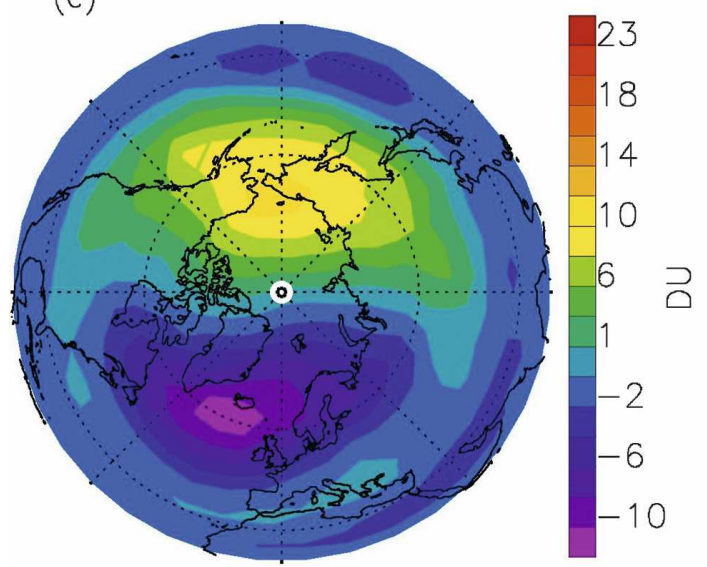

(b)

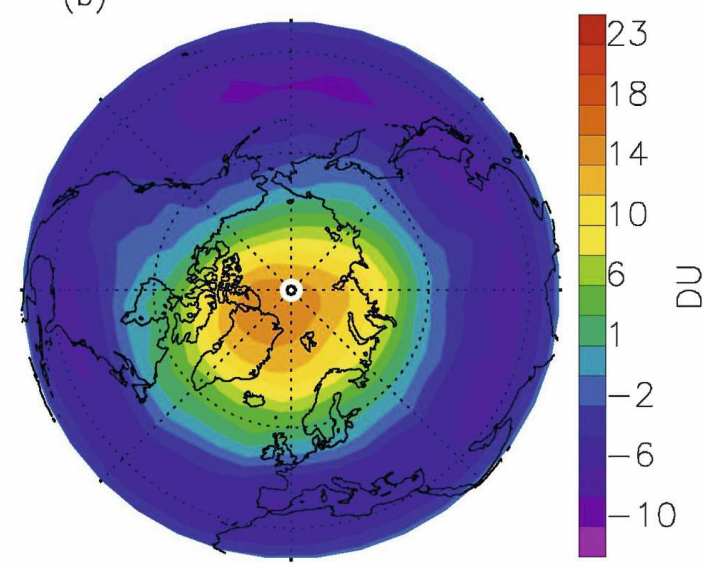

(d)

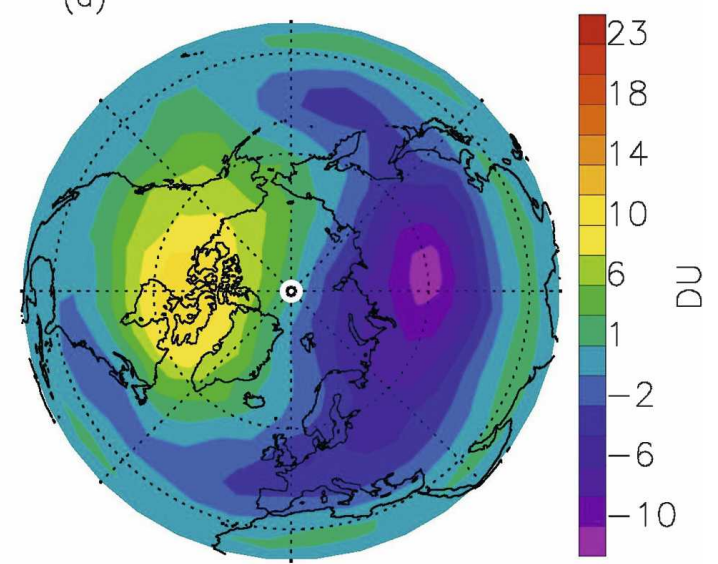

FIG. 1. The spatial patterns of the $\mathrm{O}_{3}$ anomalies regressed upon the leading PCs in the NH. Units are DU. The first four modes explain $30.4,15.9,7.1$, and $6.5 \%$ of the total variance, respectively.

these two events, which suggests the occurrence of ozone depletion after the volcano eruptions in the NH. These results are consistent with the results of Zerefos et al. (1994) and Coffey (1996).

The power spectrum of the detrended PC1 (Fig. 2b) reveals a $5 \%$ significant peak at $3.5 \mathrm{yr}$ (42 months; labeled $\mathrm{P})$. The other $5 \%$ significant spectral peaks are attributed to the QBO (28 months; Q), the QBO-AB (21 months; $\mathrm{Q}_{1}$ ) (Camp et al. 2003; Tung and Yang 1994a,b), the residual annual cycle (A), and the semiannual oscillation (6 months, $A_{1}$ ) (Gray and Pyle 1986). Because the annual cycle has a broad power spectrum, there is some residual annual cycle left after removing the monthly mean from the data. The statistical significance of the power spectral signal is obtained by comparing the amplitude of a spectral peak to the red noise spectrum (see appendix for details). Because the spatial pattern of the first mode (Fig. 1a) is positive, the negative trend in PC1 (Fig. 2a) represents a decreasing trend in ozone. The secular variation in PC1 from 1979 to
1997 displays the declining trend in ozone reported by Fusco and Salby (1999; see their Fig. 1) and chapter 3 of the WMO report (WMO 2003). However, we note that the trend has been absent since 1997 . The warmer winter of 1998/99 would contribute to the upward trend in the PC1 in the later years (Newman et al. 1997; WMO 2003).

The spectral power of the detrended PC2 is shown in Fig. 2d. It reveals 5\% significant peaks related to the QBO-AB $\left(\mathrm{Q}_{1}\right)$, the biennial oscillation (B), and the semiannual oscillation $\left(\mathrm{A}_{1}\right)$. A peak is also apparent around 8 months $\left(\mathrm{Q}_{1}^{\prime}\right)$, arising similarly from the interaction between QBO and the annual cycle (Tung and Yang 1994a; Jiang et al. 2005). The biennial oscillation (BO) has been identified in stratospheric potential vorticity by Baldwin and Dunkerton (1998). Scott and Haynes (1998) found that the BO existed in their dynamical model as alternately disturbed and quiescent winters.

To confirm the P-mode signal in Fig. 2b, we analyzed 

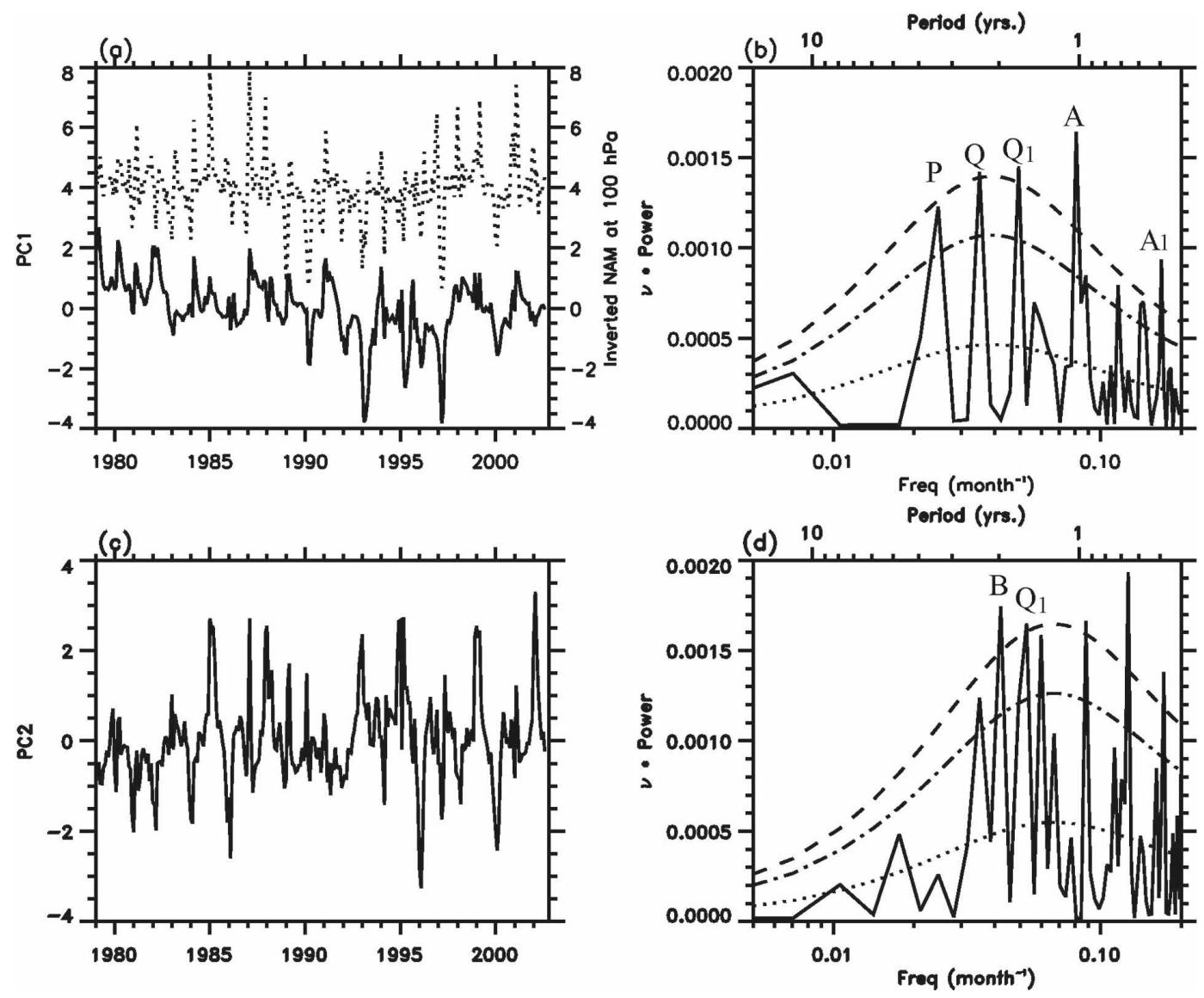

FIG. 2. (a) $\mathrm{O}_{3} \mathrm{PC} 1$ (solid) and inverted NAM index at $100 \mathrm{hPa}$ in the $\mathrm{NH}$ (dotted) shifted upward by 4 units. The correlation is 0.53 ( $0.1 \%$ significance level). (b) Power spectral estimate of detrended PC1 (solid), red noise spectrum (dotted), and $10 \%$ and $5 \%$ significance level (dashed); P, Q, $\mathrm{Q}_{1}, \mathrm{~A}$, and $\mathrm{A}_{1}$ denote the 3.5-yr signal, QBO, QBO-AB, residue seasonal cycle, and SAO respectively. (c) $\mathrm{O}_{3} \mathrm{PC} 2$ in the NH. (d) Power spectral estimate of detrended PC2 (solid), red noise spectrum (dotted), and 10\% and 5\% significance level (dashed); B denotes the BO.

the Arosa monthly mean column $\mathrm{O}_{3}$, which is the longest available $\mathrm{O}_{3}$ record (from August 1931 to December 2002). Figure 3a shows Arosa monthly mean column $\mathrm{O}_{3}$ data and third-order polynomial trend. The deseasonalized and detrended monthly $\mathrm{O}_{3}$ anomalies are shown in Fig. 3b. The power spectrum of Fig. 3b and a statistical significance test of the peaks are given in Fig. 3c. The 3.5-yr and 20 -month signals are within $1 \%$ significance level. The decadal cycle is within $5 \%$ significance level. The mechanism for the 3.5-yr signal is currently not known and will be investigated in the future.

The time series of PC3 and PC4 in the $\mathrm{NH}$ are shown in Figs. 4a,c. The corresponding power spectra (Figs. $4 b, d)$ reveal that most variability occurs over short periods (less than a year), associated with internal variability. There is evidence for a signal at the decadal period in PC3. The maxima and minima of modes 3 and
4 are stationary, as seen by the histogram for the longitudes at which the maxima of the combined pattern for modes 3 and 4 - that is, $p_{3}(t) C_{3}(\theta, \varphi)+p_{4}(t) C_{4}(\theta$, $\varphi)$-occur (Fig. 4e). Note that the preferred longitudes are $95^{\circ}, 185^{\circ}, 275^{\circ}$, and $335^{\circ} \mathrm{E}$, approximately coinciding with the nodal points in modes 3 and 4 in Figs. 1c,d. This relationship suggests that the wobbling or displacement motion is driven by planetary waves associated with topography and land-sea thermal contrasts in the NH. Episodic strong upward bursts of stationary wave activity and interference with traveling planetary waves can conceivably produce a strong displacement of the polar vortex core, which subsequently wobbles (McIntyre 1982; Salby and Garcia 1987). The displaced vortex about the pole can retain its identity for an extended period of time, giving rise to column ozone anomalies depicted in modes $3-4$ and possibly sudden stratospheric warmings (SSWs; Leovy et al. 1985). 

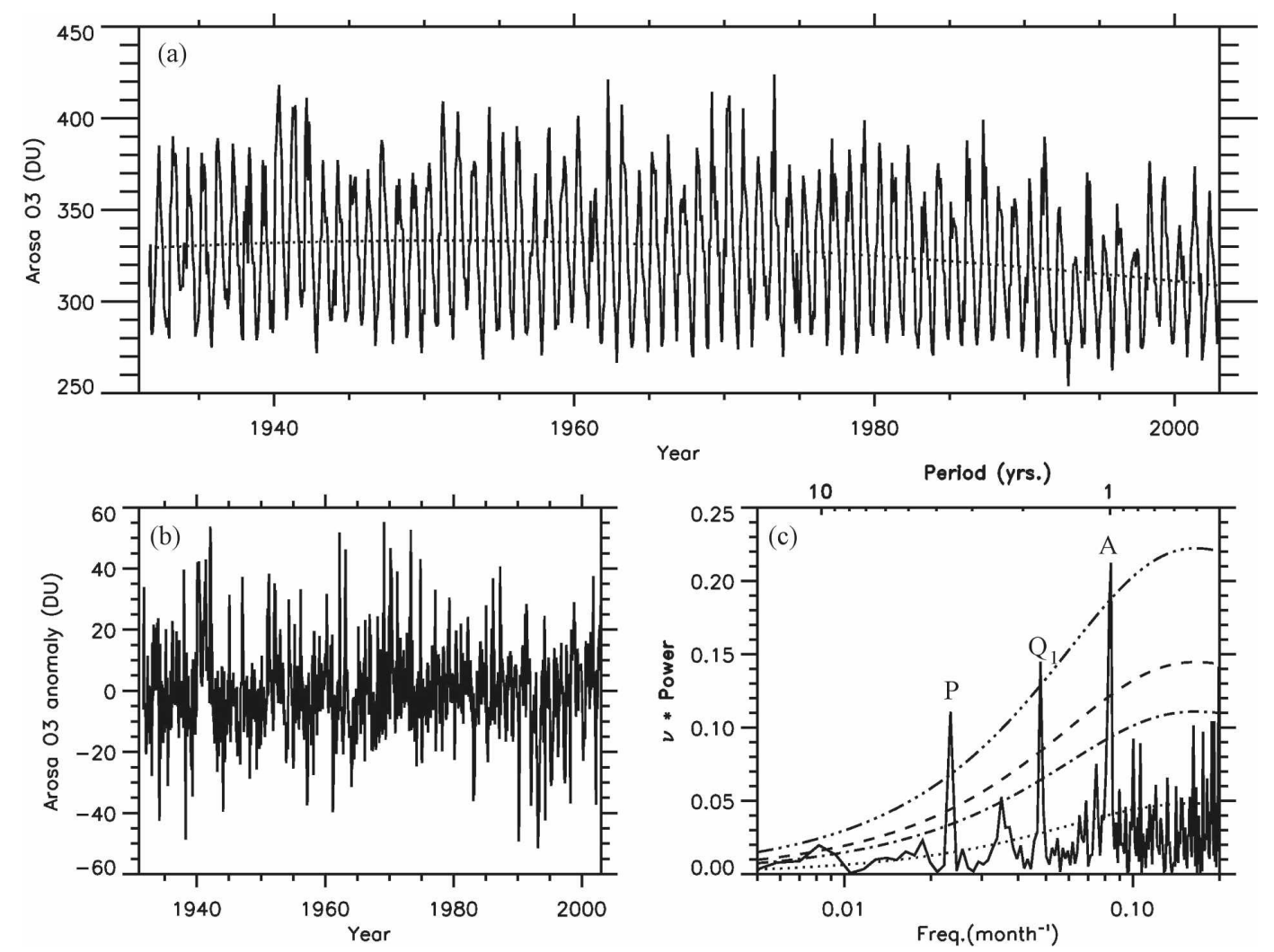

FIG. 3. (a) Arosa $\mathrm{O}_{3}$ (solid) from August 1931 to December 2002 and trend (dotted). Units are DU. (b) Deseasonalized and detrended Arosa $\mathrm{O}_{3}$ anomaly with same time period and units as (a). (c) Power spectrum of deseasonalized and detrended Arosa $\mathrm{O}_{3}$ anomaly (solid), red noise spectrum (dotted), and 10\%, 5\%, and 1\% significance level (dashed). Arosa $\mathrm{O}_{3}$ is at $46.8^{\circ} \mathrm{N}, 9.7^{\circ} \mathrm{E}$.

\section{b. Results for Northern Hemispheric GPT}

To elucidate the dynamical structure in the stratosphere, similar analyses are performed on the 30-100$\mathrm{hPa}$ GPT layer in the NCEP2 analyses. As discussed in Camp et al. (2003), the 30-hPa level is near the region of maximum ozone mixing ratio. Because ozone is the principal absorber of solar UV radiation in the stratosphere, an increase in the ozone concentration would give rise to the stratospheric heating rate and temperature. Near $100 \mathrm{hPa}$, the temperature variations are dynamically controlled and are expected to be insensitive to ozone heating. However, near $30 \mathrm{hPa}$ the temperature is partly controlled by radiative heating. Therefore increasing (decreasing) the ozone amount in the lower stratosphere will induce thermal expansion (contraction) of the air column between 30 and $100 \mathrm{hPa}$ (See section 5.2 in Part II for the quantitative relation). As presented later, thickness variations are probably caused by a combination of the direct effect by dynamical processes and an indirect effect by chemical species such as ozone as an intermediary (Camp et al. 2003).
Figure 5 shows the spatial patterns of the first four leading modes of the NCEP2 GPT data from $10^{\circ} \mathrm{N}$ to $90^{\circ} \mathrm{N}$. These four modes capture over $78 \%$ of the variance (Table 1). (The polar projection, cylindrical projection, and zonal mean of the regression coefficients are shown in Fig. A2 in the appendix.) The first, third, and fourth modes have similar structures to the first, third, and fourth ozone modes in the NH (Fig. 1). As in the ozone analyses, the first mode (Fig. 5a) is related to the NAM mode: it describes $32.3 \%$ of the total variance and is positive in the polar region and negative in the subtropics. The change of sign occurs at about $60^{\circ} \mathrm{N}$. In contrast, the first mode for the $\mathrm{O}_{3}$ column (Fig. 1a) does not change sign. Due to the increase in chlorine, the $\mathrm{O}_{3}$ column decreases at all latitudes, although there is more depletion in the polar region. Hence, EOF1 does not change sign. On the other hand, the change in GPT is part of the Brewer-Dobson circulation. An increase in upwelling in the tropics is accompanied by compensatory downwelling in the polar region, which leads to a dipole pattern in GPT. The third mode and fourth mode capture $15 \%$ and $12.4 \%$, respec- 

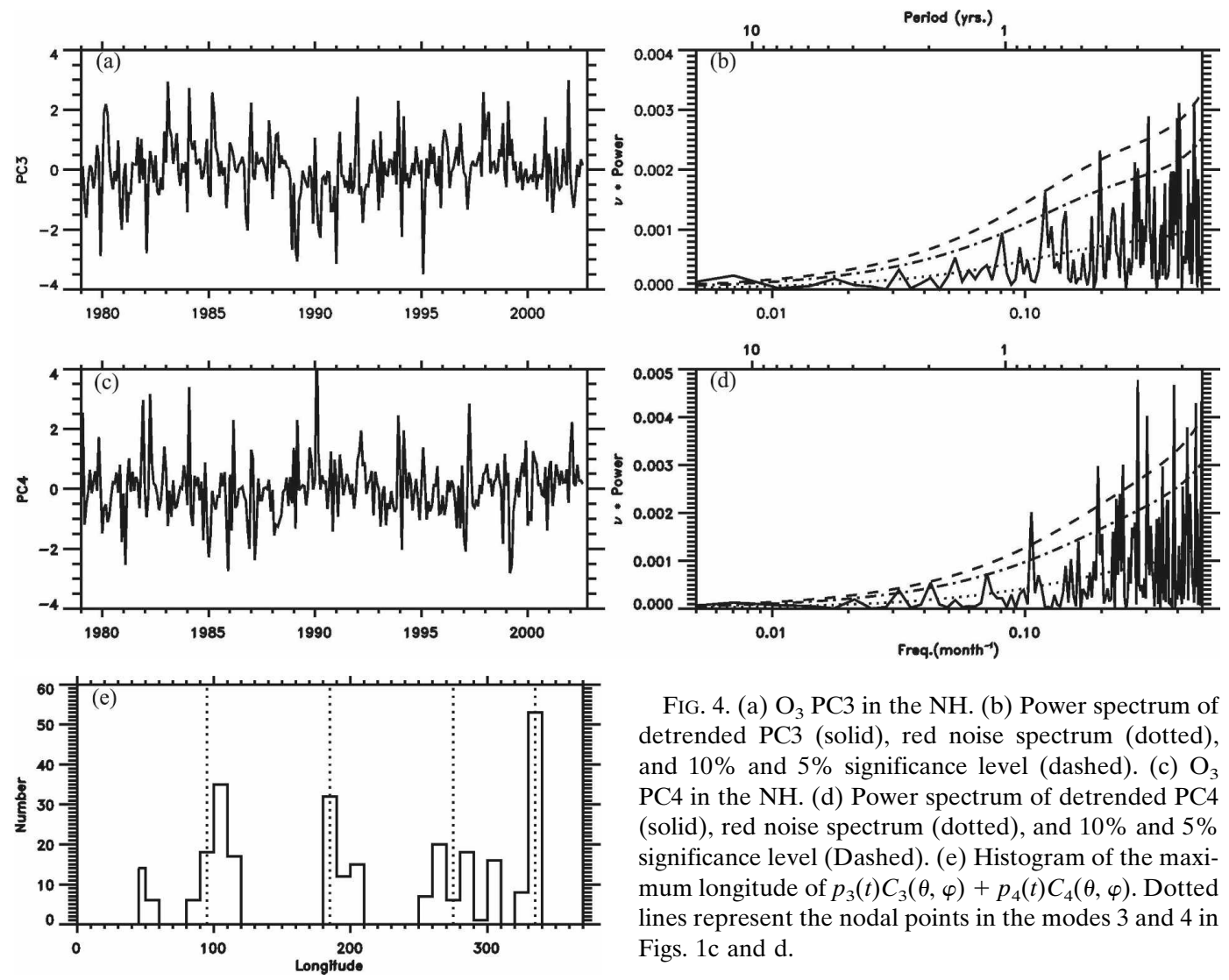

FIG. 4. (a) $\mathrm{O}_{3} \mathrm{PC} 3$ in the NH. (b) Power spectrum of detrended PC3 (solid), red noise spectrum (dotted), and $10 \%$ and $5 \%$ significance level (dashed). (c) $\mathrm{O}_{3}$ PC4 in the NH. (d) Power spectrum of detrended PC4 (solid), red noise spectrum (dotted), and $10 \%$ and $5 \%$ significance level (Dashed). (e) Histogram of the maximum longitude of $p_{3}(t) C_{3}(\theta, \varphi)+p_{4}(t) C_{4}(\theta, \varphi)$. Dotted lines represent the nodal points in the modes 3 and 4 in Figs. 1c and d.

tively, of the total variance, which are larger than the variances for the ozone field. They represent the wavenumber-1 component in the layer thickness. The centers for the wavenumber-1 component in GPT are shifted about $30^{\circ}$ compared with that in $\mathrm{O}_{3}$ column. We found out that there is a tilting for the GPT wavenumber- 1 centers from the lower altitudes to high altitudes (as shown in Fig A3). The shift between the GPT and $\mathrm{O}_{3}$ column reflects the westward tilting of the Rossby wave (e.g., Hirooka and Hirota 1985; Pawson and Kubitz 1996). In the time domain, PC1, PC3, and PC4 from the layer thickness have good correlation with the corresponding PCs in column ozone, with correlation coefficients of $0.49,0.79$, and 0.71 , respectively. The statistical significance is within $1 \%$ for all three cases. The relationships between ozone and GPT are better for modes 3 and 4 than modes 1 and 2 . The main reason is that modes 3 and 4 are intraseasonal planetary wave variations, with a more direct relationship between the locations of ozone maxima downstream of anticyclones. The spectrum of the detrended PC1 (Fig. 6b) is more dominant in the high frequencies as compared with ozone PC1 (Fig. 2b). However, the GPT PC1 (Fig. 6b) also shows a peak at $3.5 \mathrm{yr}$, with a relatively weaker

signal than the ozone PC1 (Fig. 2b). Figures $6 \mathrm{f}$ and $6 \mathrm{~h}$ show the spectra of detrended PC3 and PC4. Most signals are at high frequencies as in the ozone case (Figs. $4 \mathrm{~b}$ and $4 \mathrm{~d})$.

The second mode of the layer thickness is different from the second mode in the ozone. The GPT anomalies in the NH (Fig. 5b) are positive in almost all regions, except for a small negative area over northern Europe. The latter is not statistically significant. In contrast, the spatial pattern of the second mode of $\mathrm{O}_{3}$ column (Fig. 1b) is approximately symmetric around the pole. The spectral estimate of the PC2 (Fig. 6d) indicates peaks around 2, 4-5, and $8 \mathrm{yr}$. Although the biennial oscillation (B) appears here and in $\mathrm{PC} 2$ of the $\mathrm{O}_{3}$ column (Fig. 2d), more power resides in the lowfrequency peaks. Although the explanation for this difference is beyond the scope of this work, we speculate that $\mathrm{O}_{3}$ and GPT are sensitive to different physical and chemical processes. For example, a GPT anomaly can be forced by stationary topographical waves (see Lin 1982 and chapter 5 and Fig. 5.6 in Andrews et al. 1987). The trend for the GPT second mode is possibly related to the stratospheric cooling by the greenhouse effect (X. Jiang et al. 2008, unpublished manuscript). On the 
(a)

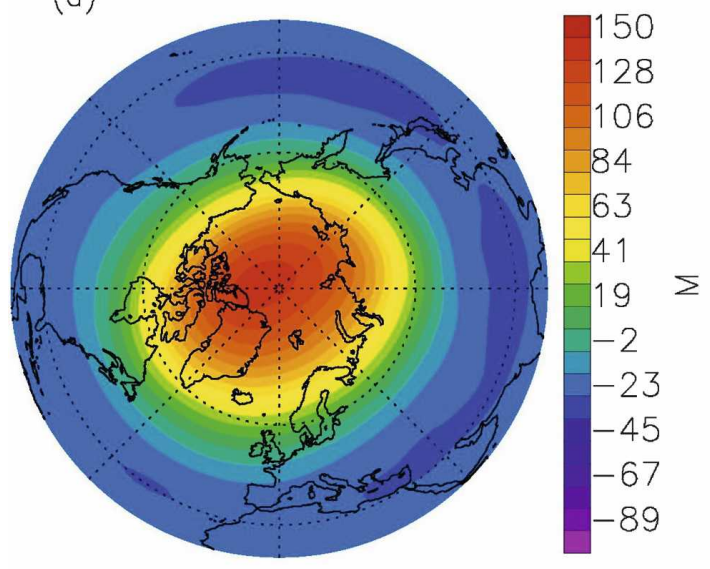

(c)

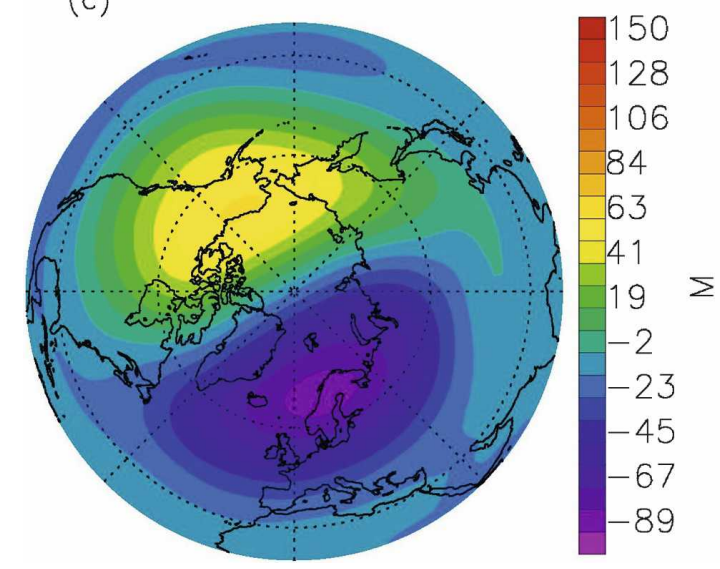

(b)

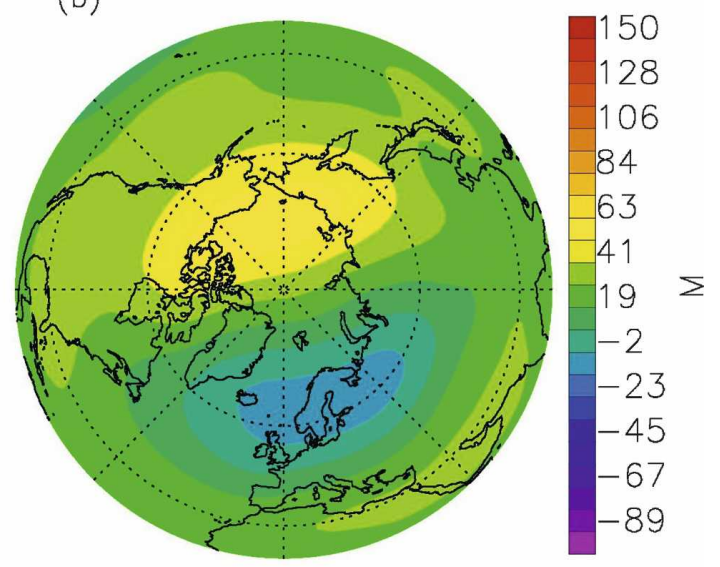

(d)

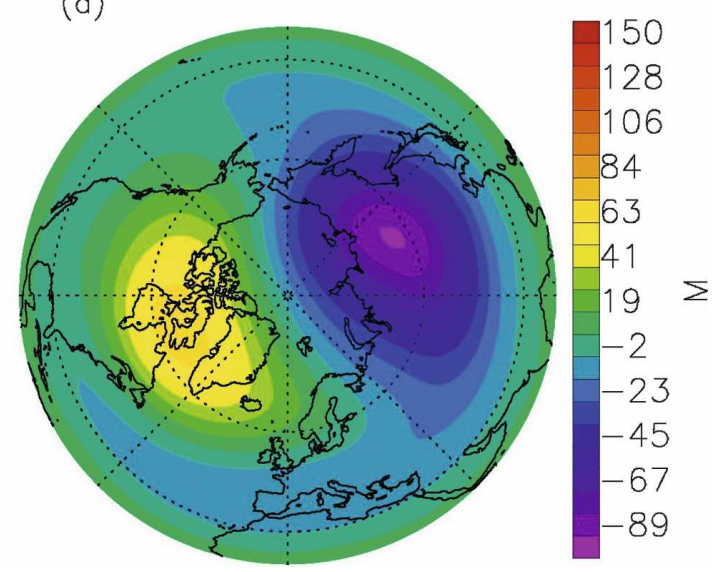

FIG. 5. The spatial patterns of the 30- to 100-hPa layer thickness anomalies regressed upon the leading PCs in the NH. Units are meters. The first four modes explain $32.3 \%, 18.5 \%, 15 \%$, and $12.4 \%$ of the total variance, respectively.

other hand, $\mathrm{O}_{3}$ is preferentially destroyed by catalytic chemistry when the polar vortex gets unusually cold (Rex et al. 2004).

\section{IAV and trends of column $\mathrm{O}_{3}$ in the GEOS-CCM}

\section{a. IAV of model column $\mathrm{O}_{3}$}

A similar analysis is now applied to the column $\mathrm{O}_{3}$ in the GEOS-CCM. Neither the solar cycle nor volcanic aerosol variations were included in the model, and it does not capture a QBO (see Horinouchi et al. 2003), so these sources of ozone variability are all absent from the simulation.

The GEOS-CCM simulation spans January 1951 to December 2004. For comparison, we select the same period as that in the combined MOD and ECMWF column $\mathrm{O}_{3}$. The first mode for the model $\mathrm{O}_{3}$ in the $\mathrm{NH}$ captures $31.8 \%$ of the total variance. The spatial pattern of the first mode in the $\mathrm{NH}$ and the associated PC1 time series are shown in Figs. 7a,b. Being zonally symmetric, the pattern is positive in sign over the whole region, which is similar to result of the combined MOD and ECMWF column $\mathrm{O}_{3}$. The correlation coefficient between detrended $\mathrm{PC} 1$ of model $\mathrm{O}_{3}$ and detrended PC1 of model $30-100-\mathrm{hPa}$ GPT is 0.62 . The trend for the $\mathrm{PC} 1$ of the model $\mathrm{O}_{3}$ in the $\mathrm{NH}$ is $-0.092 \pm 0.042$ $\mathrm{yr}^{-1}$, which is larger than the trend for the $\mathrm{PC} 1$ $\left(-0.056 \pm 0.042 \mathrm{yr}^{-1}\right)$ in the combined $\mathrm{O}_{3}$. The main reason for this is that after 1997 there was less ozone depletion in the real atmosphere than in the middle 1990s, when the stratospheric winters were cold and the polar vortex largely undisturbed (e.g., Pawson and Naujokat 1999; Manney et al. 2005). The model simulation did not reproduce this behavior, so the decreasing trend continues. The reason for this difference could be due to the misalignment of random IAV in the model compared to observations (e.g., Butchart et al. 2000) or to the absence of low-frequency forcing mechanisms (such as the solar cycle) in the model. The 

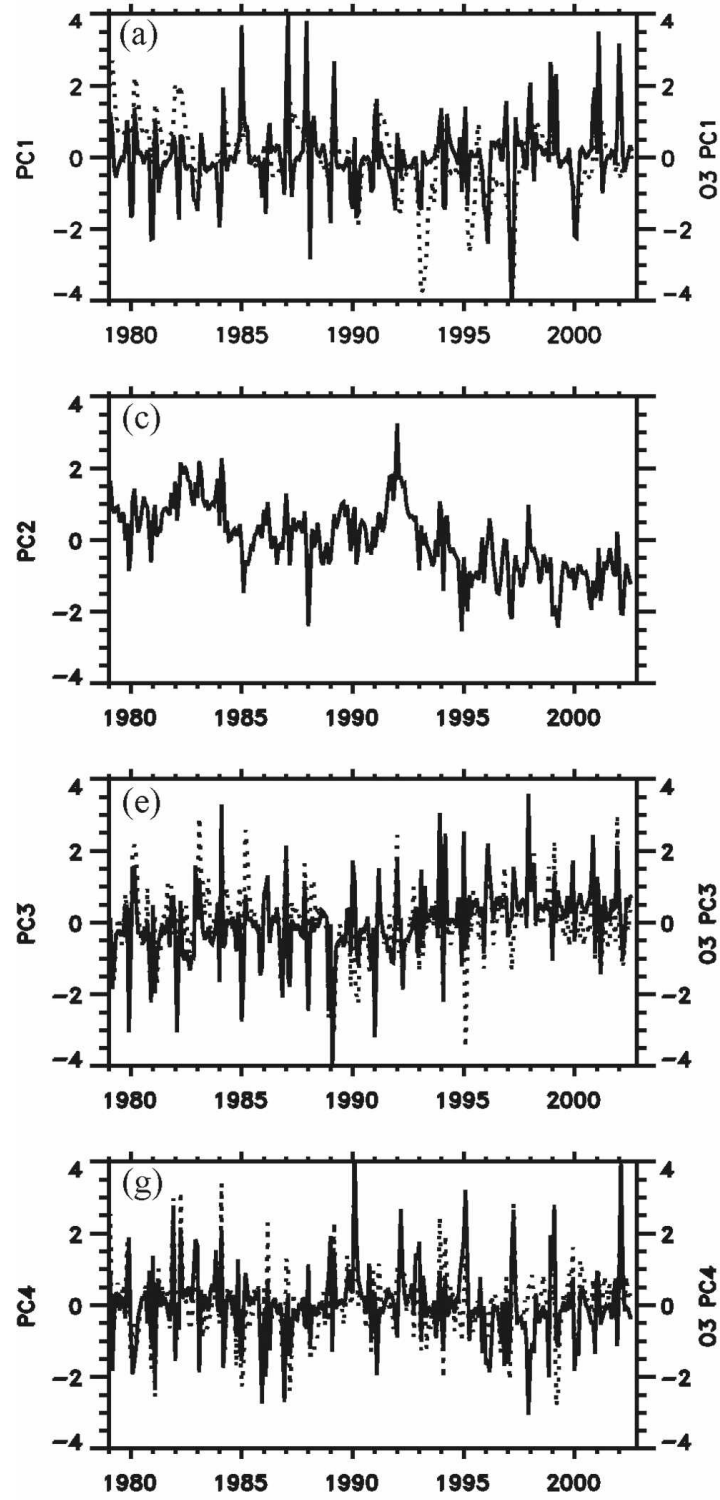
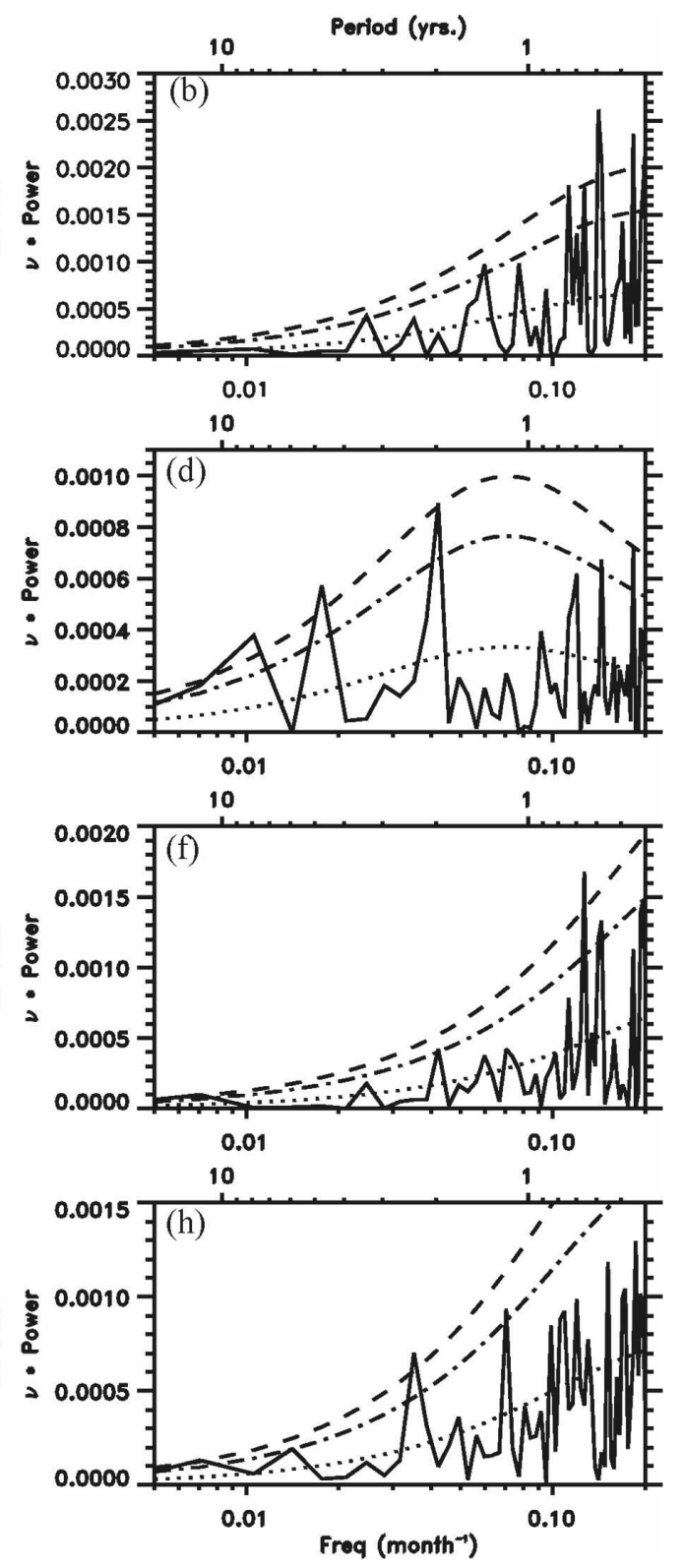

FIG. 6. (a) Layer thickness PC1 (solid) and $\mathrm{O}_{3} \mathrm{PC} 1$ (dotted) in the NH. The correlation is $0.49(0.1 \%$ significance level). (b) Power spectral estimate of detrended PC1 (solid), red noise spectrum (dotted), and $10 \%$ and $5 \%$ significance level (dashed). (c), (d) As in (a), (b), but for PC2. (e), (f) As in (a), (b), but for PC3; correlation is 0.79. (g), (h) As in (a), (b), but for PC4; correlation is 0.71 .

second, third, and fourth modes for the model $\mathrm{O}_{3}$ in the $\mathrm{NH}$ capture $9.1 \%, 7.8 \%$, and $6.0 \%$ of the total variances, respectively. The spatial patterns for these modes are similar to those in the combined $\mathrm{O}_{3}$ data in the $\mathrm{NH}$.

\section{b. Comparison of column $\mathrm{O}_{3}$ data and model trends}

To investigate the latitudinal distribution of the column $\mathrm{O}_{3}$ trends, we first calculate the zonal mean for the first $\mathrm{EOF}$ of the column ozone in the $\mathrm{NH}$. We then multiply the zonal mean value by the trend of the PC1 in the NH. The trend distribution for zonal mean of mode 1 in the $\mathrm{NH}$ is shown as a black line in Fig. 8. A trend for the ozone in the tropics is nearly absent whereas the ozone trend is large in the high latitudes [ -1 Dobson Unit (DU) $\left.\mathrm{yr}^{-1}\right]$. For comparison, we also apply the empirical mode decomposition technique (Huang et al. 1998) to the zonal mean column $\mathrm{O}_{3}$ data in the NH. This method is especially designed for sepa- 

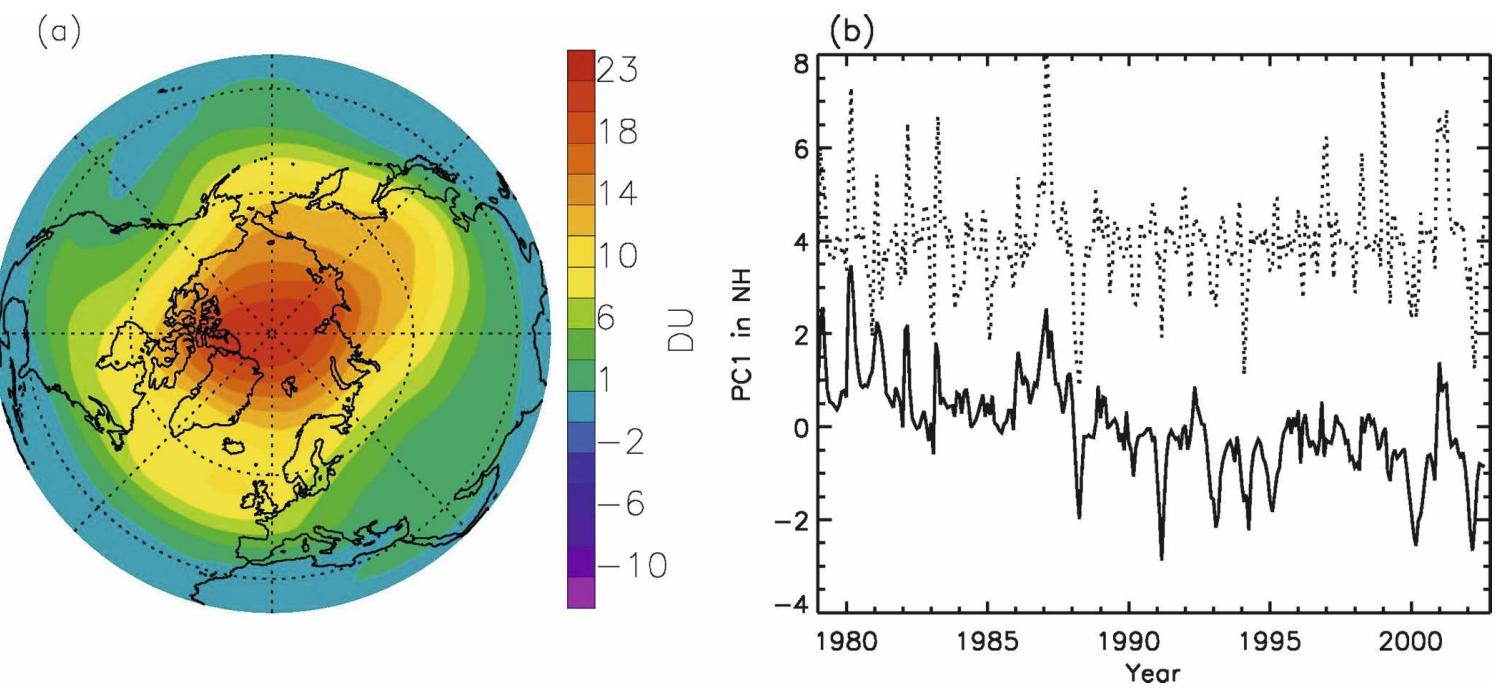

FIG. 7. (a) The spatial pattern of the model $\mathrm{O}_{3}$ anomalies regressed upon the leading PC1 in the NH. Units are DU. The first mode explains $31.8 \%$ of the total variance. (b) PC1 of the model $\mathrm{O}_{3}$ (solid line) and PC1 of the 30-100-hPa model GPT (dotted line) in the NH.

rating trend from nonstationary and nonlinear time series. After the long-term trend mode is separated from other signals, we calculate the linear trend from this mode. The trends derived from EMD method are plotted (in diamonds) in Fig. 8. Trends derived from EMD agree well with the trend derived from the first mode of column $\mathrm{O}_{3}$. The decreasing trend derived from the first mode of GEOS-CCM O 3 (the red line in Fig. 8a) is larger than the corresponding trend computed from observed ozone. This is consistent with the similarity of the spatial patterns of the leading EOFs computed from observations and the GEOS-CCM, along with the differences in dynamical variability in the last years of the analysis. The latitudinal distribution of trend for column $\mathrm{O}_{3}$ (black line and diamonds in Fig. 8) generally concur with those in Randel and Wu (2007). It also agrees with the trend derived from TOMS-SBUV for $60^{\circ} \mathrm{S}-60^{\circ} \mathrm{N}$ and those from the Stratospheric Aerosol and Gas Experiment (SAGE) sondes in the polar region as shown in Randel and $\mathrm{Wu}$ (2007).

\section{Discussion}

\section{a. $\mathrm{O}_{3}$ trend and the first mode}

We will give a heuristic explanation of why the $\mathrm{O}_{3}$ trend projects mainly into the first mode. Physical processes that control IAV in the stratosphere exhibit different symmetry properties, allowing for their separation. The first mode is nearly zonally symmetric; its amplitude increases from the equator to the pole, which is consistent with the depletion of total column $\mathrm{O}_{3}$ be- ing dominated by heterogeneous chemistry in the spring polar stratosphere (Austin et al. 2000). Averaged over a long time, the chemical loss rates are zonally symmetric. This allows a natural separation of the IAV into a largely chemically driven trend and a dynamically driven variability.

\section{b. Solar cycle and ENSO signals}

A weak decadal signal in $\mathrm{PC} 1$ and $\mathrm{PC} 3$ for $\mathrm{O}_{3}$ column is present in the $\mathrm{NH}$ (see Figs. 2b,4b); a weak signal may be present in PC3 in the SH (see Fig. 3b in Part II). The ENSO signal in the $\mathrm{O}_{3}$ column is also weak, unless we can associate the P mode (3.5-yr) with ENSO, which

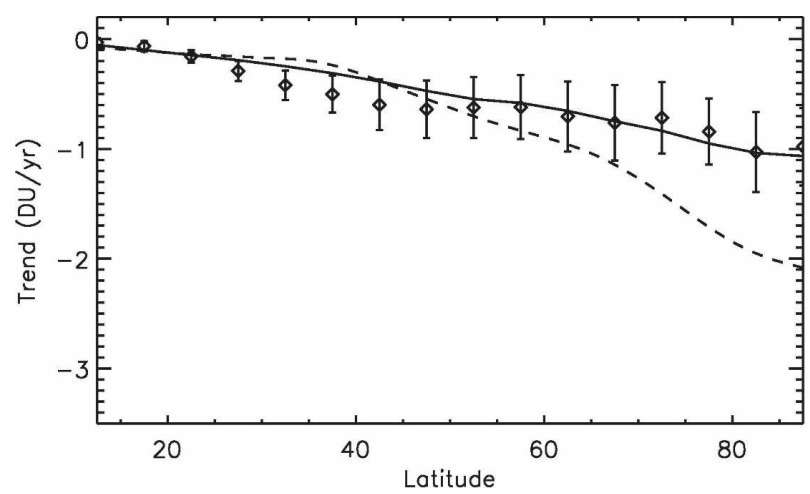

FIG. 8. Latitudinal distribution of the trend for the first mode (solid) derived from column $\mathrm{O}_{3}$ in the NH. Diamonds with error bars are the latitudinal distribution of the trend for the zonal mean column $\mathrm{O}_{3}$ derived from EMD method. The dashed line is the trend for the first mode from model $\mathrm{O}_{3}$ in the $\mathrm{NH}$. Units are DU per year. 

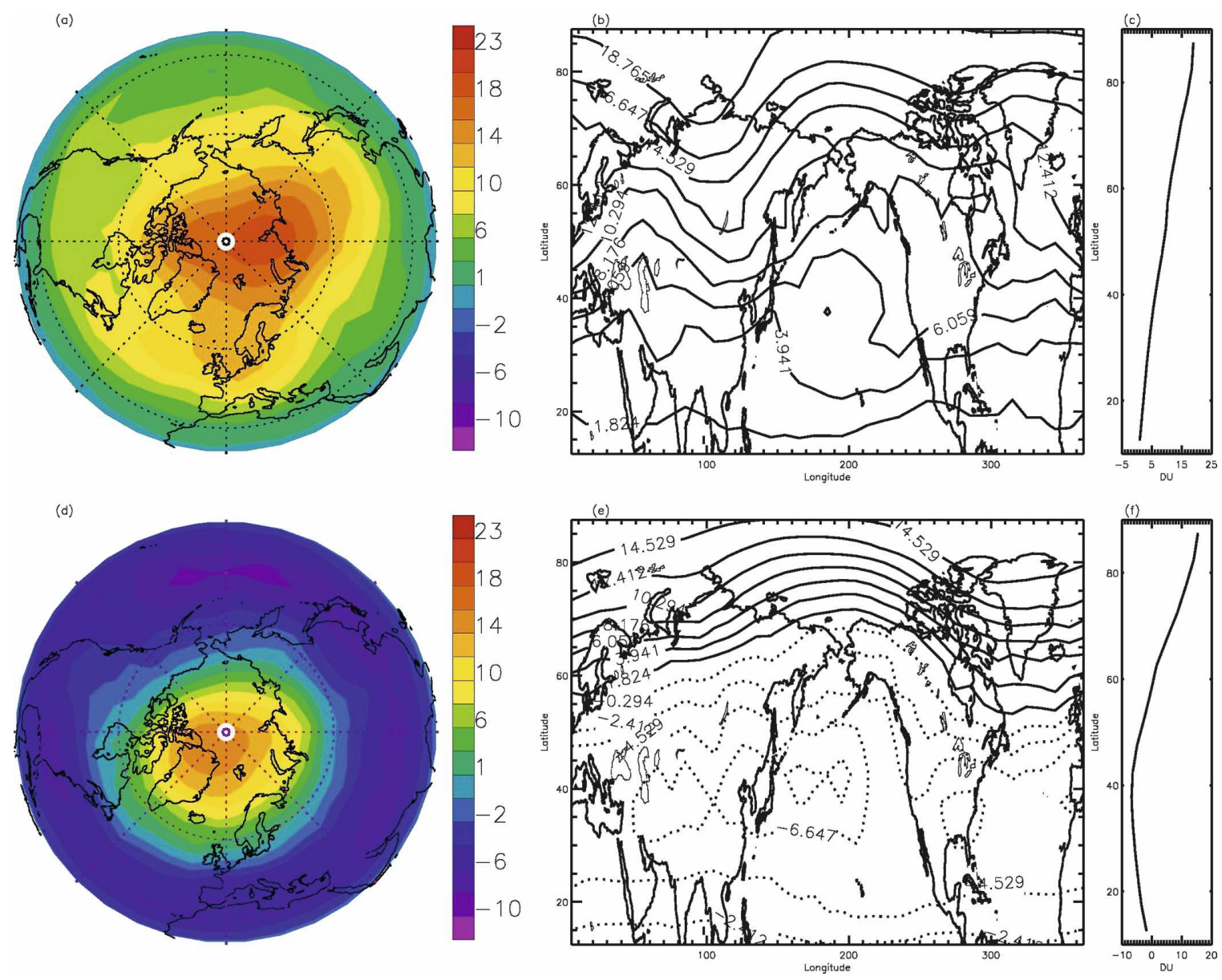

FIG. A1. The (a), (b), (c) first and (d), (e), (f) second modes of the combined MOD and ECMWF $\mathrm{O}_{3}$ anomalies in the NH. (a), (d) Polar projection maps; (b), (e) cylindrical projection maps; (c), (f) meridional profile of zonally averaged modes.

is a rather remote possibility. This characteristic is to be contrasted with the much stronger signals for solar cycle and ENSO in the tropics (Camp et al. 2003). Recently, Camp and Tung $(2007 a, b)$ found statistically significant influences of the solar cycle and ENSO in the winter polar temperature in the stratosphere. The authors attribute the cause to the possible modulation of the occurrence of sudden stratospheric warmings by the solar cycle and ENSO. Since the analysis performed in this paper used monthly averaged data, the effects of SSWs (which occurs over a few days; e.g., Limpasuvan et al. 2004) would have been minimized. A new effort with refined time resolution is needed to resolve the conflict between our results and those of Camp and Tung (2007a,b).

\section{Conclusions}

In this paper, we applied the PCA analysis to the column ozone in the $\mathrm{NH}$ high latitude for the first time.
The extensive use of PCA resolves the extratropical ozone column into zonally symmetric and asymmetric (wavenumber 1) modes (Fig. 1), with connections to the Northern Hemisphere annular modes (NAM), QBO, QBO-annual beat, biennial oscillation, semiannual oscillation, annual cycle, and solar cycle. The structures of these patterns appearing in the ozone record are similar to those in the GEOS-CCM (Fig. 7) and NCEP2 GPT (Fig. 5), although some discrepancies exist between column $\mathrm{O}_{3}$ and GPT in mode 2. The characteristics of the leading modes are summarized in Table 1 . The first symmetric mode captures about $30 \%$ of the total variance in the $\mathrm{NH}$. The negative trend in PC1 of ozone in the $\mathrm{NH}$ is partly related to the strengthening of the polar vortex in the 1980s (Fig. 2a). The trend appears to reverse itself after 1997 . The trend and the spatial pattern for the leading mode of $\mathrm{O}_{3}$ anomalies in the $\mathrm{NH}$ are well captured by the GEOS-CCM, although the trend from the model $\mathrm{O}_{3}$ is larger than that from the combined $\mathrm{O}_{3}$. 

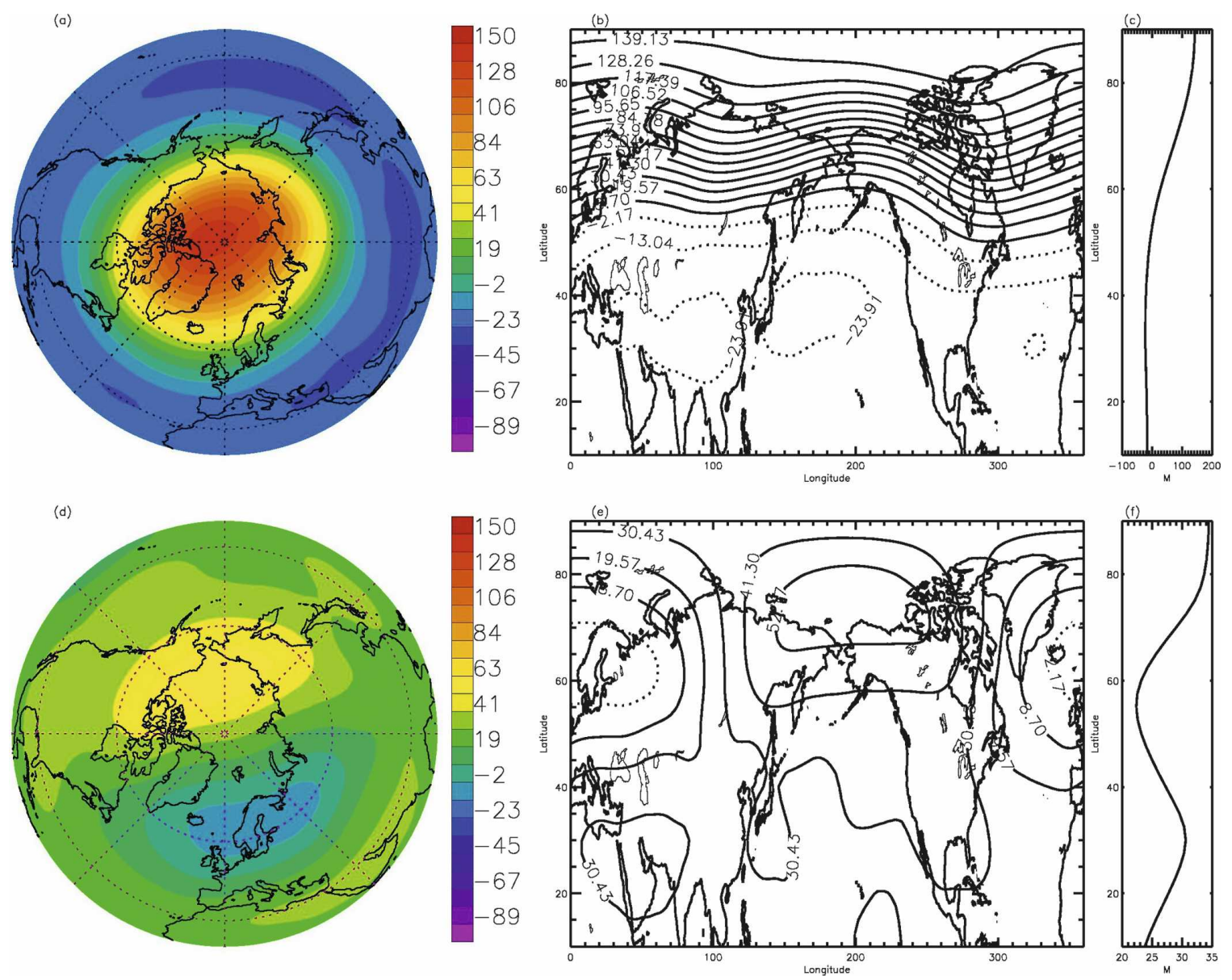

FIG. A2. The (a), (b), (c) first and (d), (e), (f) second modes of the 30- to 100-hPa layer thickness anomalies in the NH. (a), (d) polar projection maps; (b), (e) cylindrical projection maps; (c), (f) meridional profile of zonally averaged modes.

The identification of the physical processes responsible for generating the leading modes of IAV is still preliminary. Our results can be divided into two categories. In the first category, the mechanisms of the $\mathrm{QBO}, \mathrm{QBO}-\mathrm{AB}$, and the semiannual oscillation (SAO) are well known. In the second category, the physical mechanisms for the P mode signal ( $3.5 \mathrm{yr})$ in Fig. $2 \mathrm{~b}$ and biennial oscillation ( $2 \mathrm{yr}$ ) in Fig. $2 \mathrm{~d}$ are not well understood and will be investigated with the modeling study in future. The Goddard GEOS chemistry-climate model does not simulate realistically the following dynamical IAV of ozone: the QBO, QBO-AB, BO, P mode ( $3.5 \mathrm{yr}$ ), and solar cycle. In the future, the QBO and solar cycle will be included in the model, which will facilitate a more realistic comparison of variability in the model and the atmosphere.

Although there is almost no trend for the ozone in the tropics (Camp et al. 2003), the trend is large in the

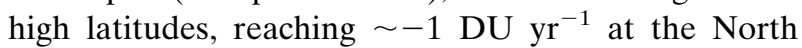
Pole (Fig. 8). To confirm these results, we apply the
EMD analysis (Huang et al. 1998) to the zonal mean column $\mathrm{O}_{3}$ data and obtain similar results. The trend in the $\mathrm{NH}$ is also reproduced by mode 1 in our analysis of the GEOS-CCM data. However, the model overestimates the decreasing ozone trends by $\sim 100 \%$ at the North Pole. In the NH, the difference in trends is dominated by discrepancies in the morphology of internally or externally forced interannual variability in the model.

Acknowledgments. We thank D. E. Waliser, M. Allen, D. Feldman, A. Ingersoll, J. Perkins, J. Weibel, M. Gerstell, and two anonymous reviewers for useful inputs and helpful comments. Special thanks are due to R. Stolarski for his contribution to the ozone simulations, $\mathrm{K}$. Jeev for deducing the missing $\mathrm{O}_{3}$ data using potential vorticity, L. M. Li for critical reading and editing of the manuscript, and R. Salawitch for improving presentation of results on $\mathrm{O}_{3}$ trends. NASA provided computational resources for running the GEOS-CCM 
(a)

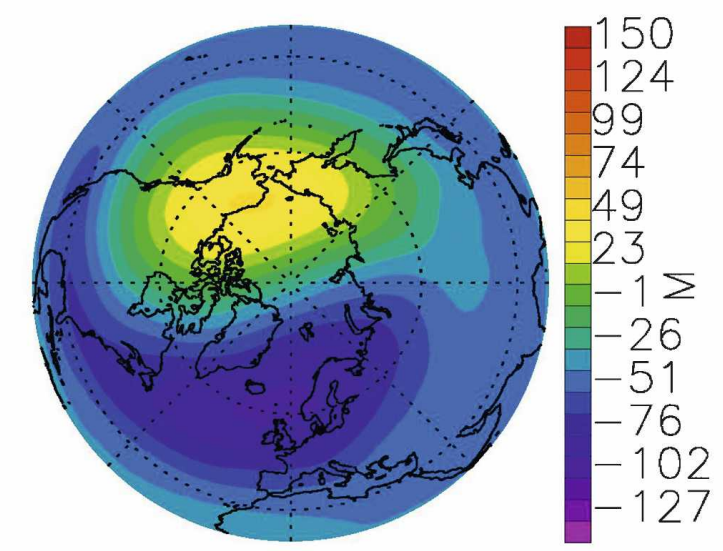

(c)

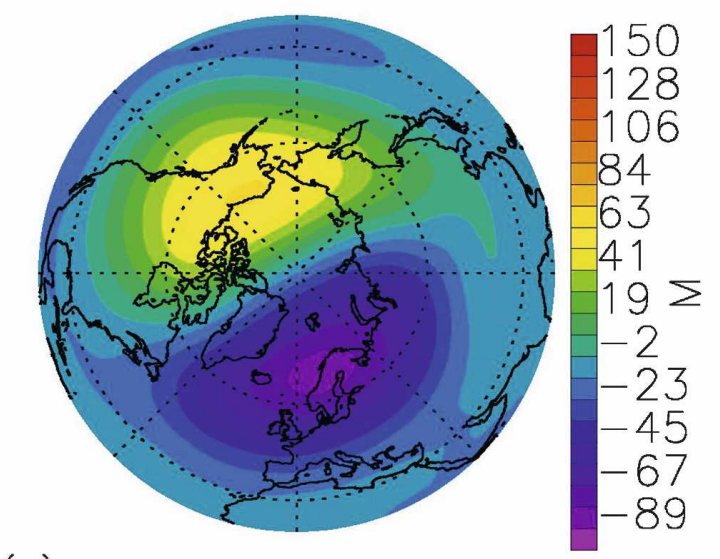

(e)

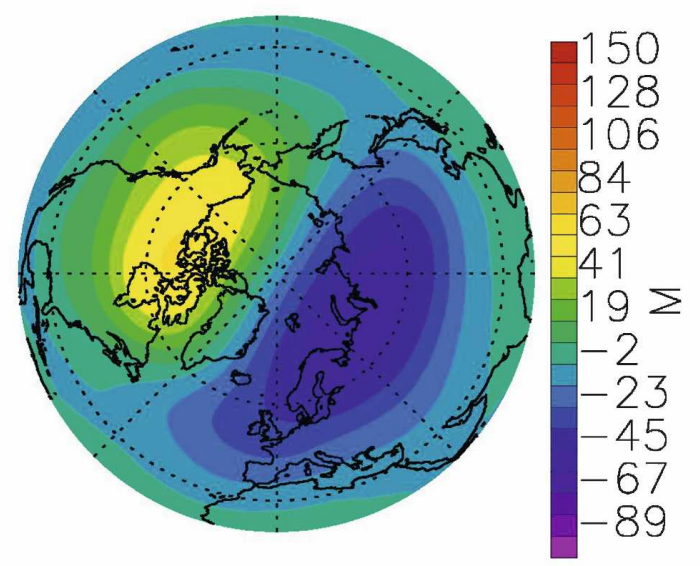

(b)

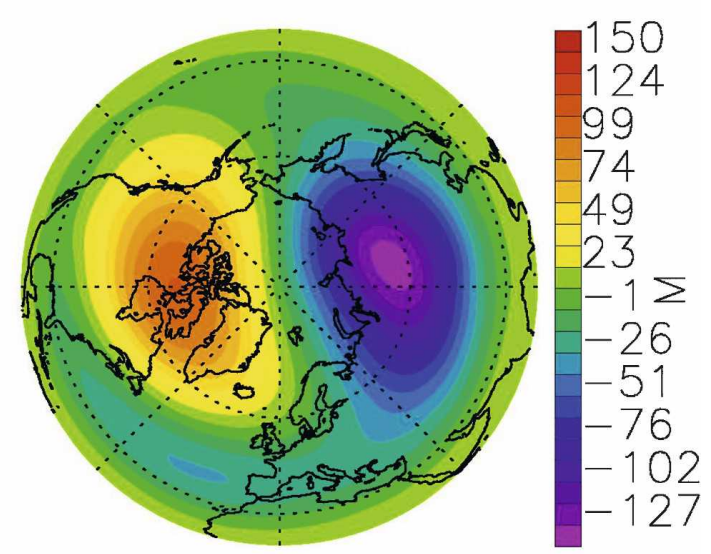

(d)

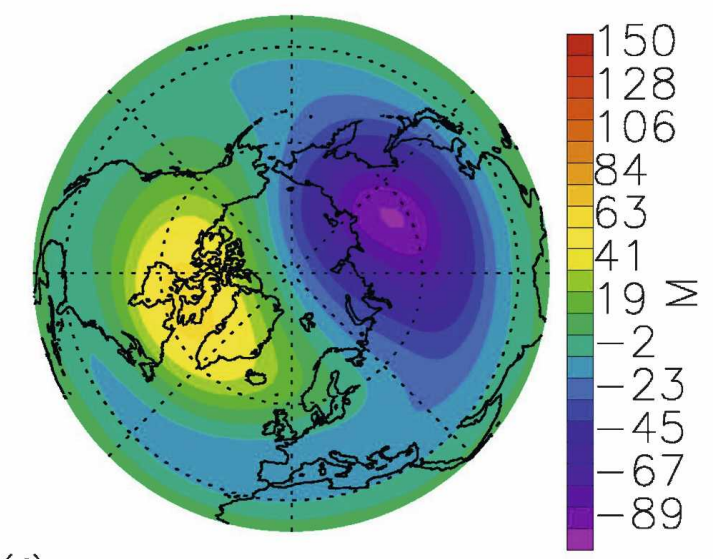

$(f)$

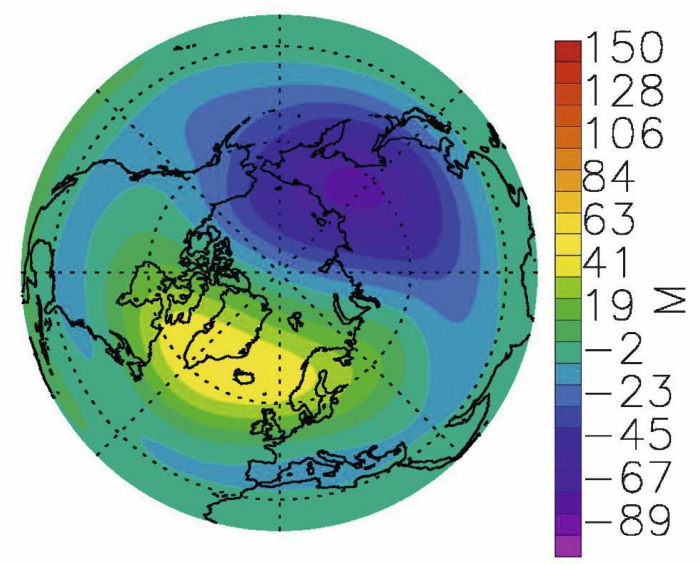

FIG. A3. The (a), (c), (e) third and (b), (d), (f) fourth modes of different layer thickness anomalies (meters) in the NH; (a), (b) 10-70, (c), (d) 30-100, and (e), (f) 50-150 hPa. 
through their high-performance computing initiative (the model was run on the "Columbia" computer at NASA Ames Research Center) This research was supported in part by NASA Grants NNG04GD76G and NNG04GN02G to the California Institute of Technology. S. Pawson and E. Nielsen were supported by NASA's Modeling and Analysis Program. V. Limpasuvan was supported by NSF Grants ATM-0213248 and ATM-0521002.

\section{APPENDIX}

\section{Statistical Significance, Cylindrical Projections, and GPT Wavenumber 1}

The appendix provides information on the statistical significance of spectral peaks in the power spectrum, cylindrical projections, zonal mean, and $t$ statistics of leading modes in the $\mathrm{NH}$ and on the tilting of wavenumber 1 in GPT.

\section{a. Statistical significance of signals in power spectrum}

The statistical significance of signals in a power spectrum is obtained by comparing the amplitude of a spectral peak to the red noise spectrum. The red noise spectrum used in constructing the null hypothesis for significance is the spectrum associated with the autocorrelation function $\rho$ (Gilman et al. 1963). Here, $\rho$ is the average of one-lag autocorrelation and the square root of the two-lag autocorrelation. The red noise spectrum associated with the autocorrelation function is

$$
L(h, \rho, M)=\frac{1-\rho^{2}}{1-2 \rho \cos \left(\frac{h \pi}{M}\right)+\rho^{2}},
$$

where $h$ is the frequency and $M$ is the maximum lag (Gilman et al. 1963). The 10\%, 5\%, and 1\% significance levels for the power spectrum are found using $F$ statistics to compare the spectrum to the red noise spectrum.

\section{b. Cylindrical projections, zonal mean, and $t$ statistics of leading modes}

The polar projection, the cylindrical projection, and the zonal mean of the leading two modes for the combined MOD and ECMWF $\mathrm{O}_{3}$ anomalies in the $\mathrm{NH}$ are shown in Fig. A1. Similar results for the 30- to $100-\mathrm{hPa}$ geopotential height layer thickness anomalies are shown in Fig. A2. The first mode of $\mathrm{O}_{3}$ anomalies in the $\mathrm{NH}$ is positive. The second mode of $\mathrm{O}_{3}$ anomalies in the $\mathrm{NH}$ has a node at approximately $60^{\circ} \mathrm{N}$. The first mode of GPT anomalies in the $\mathrm{NH}$ is positive in the polar region and negative in the subtropical region. The second mode of GPT anomalies in the NH is positive in almost all regions, except for a small negative area in northern Europe, which is not statistically significant.

The associated $t$ statistics for the regression coefficients of $\mathrm{O}_{3}$ and GPT anomalies are calculated. For the regression coefficients $\beta_{k}$, the $t$ statistic is defined by $t_{k}=\left|\beta_{k} / \operatorname{Se}\left(\beta_{k}\right)\right|$, where $\operatorname{Se}\left(\beta_{k}\right)$ is the 1-sigma uncertainty estimate for $\beta_{k}$. The result is statistically significant when $t_{k}$ is larger than a certain value $t_{0}$. Given the number of degrees of freedom, $t_{0}$ can be found from the $t$ distribution table. The number of degrees of freedom can be calculated by $N^{*}=-(N \ln \rho) / 2-5$, where $N$ is the length of the data and $\rho$ is the autocorrelation function. Because the autocorrelations are different for the different positions, $t_{0}$ with a $5 \%$ significance level varies from 1.653 to $1.734 ; t_{k}$ of $\mathrm{O}_{3}$ and GPT anomalies are larger than 1.734 in most areas. This suggests that the results are statistically significant in most areas.

\section{c. Tilting of wavenumber 1 in GPT}

The third and fourth modes for the GPT at different altitudes are shown in Fig. A3. The GPT wavenumber-1 centers tilt westward from the lower altitudes (50-150 $\mathrm{hPa})$ to high altitudes $(10-70 \mathrm{hPa})$. It is consistent with the westward phase tilting results from satellite observations in Hirooka and Hirota (1985).

\section{REFERENCES}

Andrews, D. G., J. R. Holton, and C. B. Leovy, 1987: Middle Atmosphere Dynamics. Academic Press, 489 pp.

Austin, J., J. R. Knight, and N. Butchart, 2000: Three-dimensional chemical model simulations of the ozone layer: 1979-2015. Quart. J. Roy. Meteor. Soc., 126, 1533-1556.

Baldwin, M. P., and T. J. Dunkerton, 1998: Biennial, quasibiennial, and decadal oscillations of potential vorticity in the northern stratosphere. J. Geophys. Res., 103, 3919-3928.

—, and 1999: Propagation of the Arctic Oscillation from the stratosphere to the troposphere. J. Geophys. Res., 104, 30 937-30 946.

— D. B. Stephenson, I. T. Jolliffe, 2008: Spatial weighting and iterative projection methods for EOFs. J. Climate, in press.

Butchart, N., J. A. Ustin, J. R. Knight, A. A. Scaife, and M. L. Gallani, 2000: The response of the stratospheric climate to projected changes in the concentrations of well-mixed greenhouse gases from 1992 to 2051. J. Climate, 13, 2142-2159.

Camp, C. D., and K.-K. Tung, 2007a: The influence of the solar cycle and $\mathrm{QBO}$ on the late-winter stratospheric polar vortex. J. Atmos. Sci., 64, 1267-1283.

$\longrightarrow$, and _ 2007b: Stratospheric polar warming by ENSO in winter: A statistical study. Geophys. Res. Lett., 34, L04809, doi:10.1029/2006GL028521.

_ M. S. Roulston, and Y. L. Yung, 2003: Temporal and spatial patterns of the interannual variability of total ozone in the tropics. J. Geophys. Res., 108, 4643, doi:10.1029/2001JD001504.

Coffey, M. T., 1996: Observations of the impact of volcanic activ- 
ity on stratospheric chemistry. J. Geophys. Res., 101, 67676780.

Da Silva, A., and Coauthors, 2005: Documentation and validation of The Goddard Earth Observation System (GEOS) data assimilation system, version 4. NASA Tech. Rep. TM-2005104606, Vol. 26, 166 pp.

Dethof, A., and E. V. Holm, 2004: Ozone assimilation in the ERA-40 reanalysis project. Quart. J. Roy. Meteor. Soc., 130, 2851-2872.

Eder, B. K., S. K. LeDuc, and J. E. Sickles, 1999: A climatology of total ozone mapping spectrometer data using rotated principal component analysis. J. Geophys. Res., 104, 3691-3709.

Fusco, A. C., and M. L. Salby, 1999: Interannual variations of total ozone and their relationship to variations of planetary wave activity. J. Climate, 12, 1619-1629.

Gilman, D. L., F. J. Fuglister, and J. M. Mitchell, 1963: On the power spectrum of "red noise." J. Atmos. Sci., 20, 182-184.

Gray, L. J., and J. A. Pyle, 1986: The semiannual oscillation and equatorial tracer distributions. Quart. J. Roy. Meteor. Soc., 112, 387-407.

Hirooka, T., and I. Hirota, 1985: Normal mode Rossby waves observed in the upper stratosphere. Part II: Second antisymmetric and symmetric modes of zonal wavenumbers 1 and 2 . J. Atmos. Sci., 42, 536-548.

Hood, L. L., and B. E. Soukharev, 2003: Quasi-decadal variability of the tropical lower stratosphere: The role of extratropical wave forcing. J. Atmos. Sci., 60, 2389-2403.

Horinouchi, T., and Coauthors, 2003: Tropical cumulus convection and upward propagating waves in middle-atmospheric GCMs. J. Atmos. Sci., 60, 2765-2782.

Huang, N. E., and Coauthors, 1998: The empirical mode decomposition and Hilbert spectrum for nonlinear and nonstationary time series analysis. Proc. Roy. Soc. London, 454, 903-995.

Jiang, X., C. D. Camp, R. Shia, D. Noone, C. Walker, and Y. L. Yung, 2004: Quasi-biennial oscillation and quasi-biennial oscillation-Annual beat in the tropical total column ozone: A two-dimensional model simulation. J. Geophys. Res., 109, D16305, doi:10.1029/2003JD004377.

- D. B. A. Jones, R. Shia, D. E. Waliser, and Y. L. Yung, 2005: Spatial patterns and mechanisms of the quasi-biennial oscillation-Annual beat of ozone. J. Geophys. Res., 110, D23308, doi:10.1029/2005JD006055.

— S. Pawson, C. D. Camp, J. E. Nielsen, R.-L. Shia, T. Liao, V. Limpasuvan, and Y. L. Yung, 2008: Interannual variability and trend of extratropical ozone. Part II: Southern Hemisphere. J. Atmos. Sci., 65, 3030-3041.

Kalnay, E., and Coauthors, 1996: The NCEP/NCAR 40-Year Reanalysis Project. Bull. Amer. Meteor. Soc., 77, 437-471.

Kistler, R., and Coauthors, 2001: The NCEP-NCAR 50-Year Reanalysis: Monthly means CD-ROM and documentation. Bull. Amer. Meteor. Soc., 82, 247-267.

Leovy, C. B., C.-R. Sun, M. H. Hitchman, E. E. Remsberg, J. M. Russell, L. L. Gordley, J. C. Gille, and L. V. Lyjak, 1985: Transport of ozone in the middle stratosphere: Evidence for planetary wave breaking. J. Atmos. Sci., 42, 230-244.

Limpasuvan, V., D. W. J. Thompson, and D. L. Hartmann, 2004: The life cycle of Northern Hemisphere sudden stratospheric warmings. J. Climate, 17, 2584-2596.

Lin, B.-D., 1982: The behavior of winter stationary planetary waves forced by topography and diabatic heating. J. Atmos. Sci., 39, 1206-1226.

Manney, G. L., H. A. Michelsen, M. L. Santee, M. R. Gunson,
F. W. Irion, A. E. Roche, and N. J. Livesey, 1999: Polar vortex dynamics during spring and fall diagnosed using trace gas observations from the Atmospheric Trace Molecule Spectroscopy instrument. J. Geophys. Res., 104, 18 841-18 866.

, K. Krüger, J. L. Sabutis, S. A. Sena, and S. Pawson, 2005: The remarkable 2003-2004 winter and other recent warm winters in the Arctic stratosphere since the late 1990s. J. Geophys. Res., 110, D04107, doi:10.1029/2004JD005367.

McIntyre, M. E., 1982: How well do we understand the dynamics of stratospheric warmings? J. Meteor. Soc. Japan, 60, 37-65.

McPeters, R., and Coauthors, 1996: Nimbus-7 Total Ozone Mapping Spectrometer (TOMS) data products user's guide. NASA Reference Publication 1384, 67 pp.

Newman, P. A., J. F. Gleason, R. D. McPeters, and R. S. Stolarski, 1997: Anomalously low ozone over the Arctic. Geophys. Res. Lett., 24, 2689-2692.

Pawson, S., and T. Kubitz, 1996: Climatology of planetary waves in the northern stratosphere. J. Geophys. Res., 101, $16987-$ 16996.

— in the northern lower stratosphere. J. Geophys. Res., 104, 14 209-14 222.

——, R. S. Stolarski, A. R. Douglass, P. A. Newman, J. E. Nielsen, S. M. Frith, and M. L. Gupta, 2008: Goddard Earth Observing System chemistry-climate model simulations of stratospheric ozone-temperature coupling between 1950 and 2005. J. Geophys. Res., 113, D12103, doi:10.1029/2007JD009511.

Preisendorfer, R. W., 1988: Principal Component Analysis in Meteorology and Oceanography. Elsevier, $425 \mathrm{pp}$.

Press, W., S. Teukolsky, W. Vetterling, and B. Flannery, 1992: Numerical Recipes in Fortran 77: The Art of Scientific Computing. Cambridge University Press, 933 pp.

Randel, W. J., and F. Wu, 2007: A stratospheric ozone profile data set for 1979-2005: Variability, trends, and comparisons with column ozone data. J. Geophys. Res., 112, D06313, doi:10.1029/2006JD007339.

,,- , and R. Stolarski, 2002: Changes in column ozone correlated with the stratospheric EP flux. J. Meteor. Soc. Japan, 80, 849-862.

Rayner, N. A., D. E. Parker, E. B. Horton, C. K. Folland, L. V. Alexander, D. P. Rowell, E. C. Kent, and A. Kaplan, 2003: Global analyses of sea surface temperature, sea ice, and night marine air temperature since the late nineteenth century. $J$. Geophys. Res., 108, 4407, doi:10.1029/2002JD002670.

Rex, M., R. J. Salawitch, P. von der Gathen, N. R. P. Harris, M. P. Chipperfield, and B. Naujokat, 2004: Arctic ozone loss and climate change. Geophys. Res. Lett., 31, L04116, doi:10.1029/ 2003 GL018844.

Richman, M. B., 1986: Rotation of principal components. J. Climatol., 6, 293-335.

Salby, M. L., and R. R. Garcia, 1987: Vacillations induced by interference of stationary and traveling planetary waves. $J$. Atmos. Sci., 44, 2679-2711.

Scott, R. K., and P. H. Haynes, 1998: Internal interannual variability of the extratropical stratospheric circulation: The lowlatitude flywheel. Quart. J. Roy. Meteor. Soc., 124, 2149-2173.

Shindell, D. T., R. L. Miller, G. A. Schmidt, and L. Pandolfo, 1999: Simulation of recent northern winter climate trends by greenhouse-gas forcing. Nature, 399, 452-455.

Simmons, A. J., A. Untch, C. Jakob, P. Kallberg, and P. Unden, 1999: Stratospheric water vapour and tropical tropopause temperatures in ECMWF analyses and multi-year simulations. Quart. J. Roy. Meteor. Soc., 125, 353-386. 
Stolarski, R. S., A. R. Douglass, M. Gupta, P. A. Newman, S. Pawson, M. R. Schoeberl, and J. E. Nielsen, 2006a: An ozone increase in the Antarctic summer stratosphere: A dynamical response to the ozone hole. Geophys. Res. Lett., 33, L21805, doi:10.1029/2006GL026820.

, - - S. E. Steenrod, and S. Pawson, 2006b: Trends in stratospheric ozone: Lessons learned from a 3D chemical transport model. J. Atmos. Sci., 63, 1028-1041.

Thompson, D. W. J., and J. M. Wallace, 2000: Annular modes in the extratropical circulation. Part I: Month-to-month variability. J. Climate, 13, 1000-1016.

Tung, K.-K., and H. Yang, 1994a: Global QBO in circulation and ozone. I. Reexamination of observational evidence. J. Atmos. Sci., 51, 2699-2707.

- and - 1994b: Global QBO in circulation and ozone. II: A simple mechanistic model. J. Atmos. Sci., 51, 2708-2721.

Uppala, S. M., and Coauthors, 2005: The ERA-40 re-analysis. Quart. J. Roy. Meteor. Soc., 131, 2961-3012.

Weatherhead, E. C., and Coauthors, 2000: Detecting the recovery of total column ozone. J. Geophys. Res., 105, 22 201-22 210.

WMO, 2003: Scientific assessment of ozone depletion: 2002. Global Ozone Research and Monitoring Project Rep. 47, 498 pp.

Zerefos, C. S., K. Tourpali, and A. F. Bais, 1994: Further studies on possible volcanic signal to the ozone layer. J. Geophys. Res., 99, 25 741-25 746. 